\title{
Spot14/Spot14R expression may be involved in MSC adipogenic differentiation in patients with adolescent idiopathic scoliosis
}

\author{
QIFEI WANG, JUNLIN YANG, XIANG LIN, ZIFANG HUANG, CHAOFAN XIE and HENGWEI FAN \\ Department of Scoliosis, The First Affiliated Hospital of Sun Yat-Sen University, \\ Guangzhou, Guangdong 510080, P.R. China
}

Received April 6, 2015; Accepted February 29, 2016

DOI: $10.3892 / \mathrm{mmr} .2016 .5109$

\begin{abstract}
The aim of the present study was to evaluate the different expression levels of thyroid hormone responsive (THRSP; Spot14)/S14 related, Mig12 (S14R) during bone marrow mesenchymal stem cell (BM-MSC) adipogenesis in adolescent idiopathic scoliosis (AIS) patients. MSCs were retrospectively isolated from AIS patients and controls, and adipogenic differentiation was induced. Total RNA was extracted for Affymetrix 3'-IVT expression profiling microarrays and compared with the results from healthy controls. The results were confirmed by semiquantitative reverse transcription-polymerase chain reaction (RT-PCR) validation and the protein expression levels of Spot14 and its paralogous gene S14R by western blotting and immunohistochemistry. A total of 300 significantly altered mRNAs were detected (111 upregulated and 189 downregulated) and confirmed by RT-qPCR. The mRNA expression levels of seven genes, including Spot14, were altered by $>2$-fold in AIS patients. Spot14/S14R was selected for further investigation. The results of the western blotting demonstrated that mRNA and protein expression levels of Spot14/S14R were significantly higher in AIS patients than the controls $(\mathrm{P}<0.05)$. Immunohistochemistry demonstrated Spot14 was expressed in $85 \%$ (17/20 cases) in adipose tissue samples from AIS patients and $23.1 \%$ (3/13 cases) of adipose tissue samples from controls. The positive ratio of Spot14 in adipose tissue samples from AIS was significantly higher than the controls $(\mathrm{P}<0.001)$. The results of the present study indicated that Spot14/S14R were differently expressed in MSC adipogenesis in AIS patients, and they may be important in the abnormal adipogenic differentiation in AIS.
\end{abstract}

Correspondence to: Professor Junlin Yang, Department of Scoliosis, The First Affiliated Hospital of Sun Yat-Sen University, 74 Zhongsan Er Road, Guangzhou, Guangdong 510080, P.R. China E-mail: yjunlinsc@gmail.com

Key words: mesenchymal stem cell, adolescent idiopathic scoliosis, adipogenesis, Spot14, microarrays

\section{Introduction}

Adolescent idiopathic scoliosis (AIS) is a complex spinal deformity that often affects female adolescents. The reported incidence of the disease is $2-4 \%$ (1-3). The pathogenesis of AIS remains to be elucidated, however, etiological and epidemiological studies have indicated that the incidence and the development of the disease involve differences in gene expression and regulation. A number of studies have reported that scoliosis patients have low bone density, and have demonstrated that idiopathic scoliosis patients exhibit abnormal osteogenesis and reduced numbers of osteogenic cells (4-7). Epidemiological studies have demonstrated that AIS patients exhibit physical characteristics, including tallness and low body mass index (BMI), which suggest that the processes of adipogenic and osteogenic metabolism may be affected in AIS patients.

Mesenchymal stem cells (MSCs) differentiate to bone, cartilage and adipose cells. AIS patients present with osteopenia, and previous studies suggest that MSCs from patients with AIS undergo abnormal differentiation during development of osteoblasts, chondrocytes and adipocytes $(8,9)$. Currently, research is focused on osteogenesis and chondrogenesis of MSCs in patients with AIS, however, interest in adipogenic differentiation is increasing (10), as osteogenic and adipogenic differentiation are opposing processes.

The current study aimed to evaluate differential gene expression between AIS patients and controls by performing microarrays of MSC adipogenic differentiation, with a further investigation using semiquantitative reverse transcription-polymerase chain reaction (RT-qPCR). Thyroid hormone-inducible nuclear protein (THRSP; Spot14) and its paralogous gene S14 related, Mig12 (S14R) were selected for further confirmation and examination by western blotting and immunohistochemistry.

\section{Materials and methods}

MSC extraction and flow cytometry. A total of 33 subjects, including 20 AIS patients (female, 13-16 years old, excluding subjects with liver, kidney, endocrine or blood diseases, infections and tumors) and 13 volunteers (female, 12-18 years old with lower extremity or pelvic fractures and no other diseases), were enrolled in the present study between September 2002 and 
January 2013 from the scoliosis center of the First Affiliated Hospital of Sun Yat-Sen University (Guangzhou, China). The present study was approved by the ethics committee of The First Affiliated Hospital of Sun Yat-Sen University. Written informed consent was obtained from all subjects and their parents (in the case of children $<16$ ) prior to entering the present study.

Bone marrow samples $(10 \mathrm{ml})$ were pre-operatively collected with a bone marrow biopsy needle via the posterior superior iliac crest following general anesthesia. Percoll $\left(1.073 \times 10^{-3} \mathrm{~g} / 1\right.$, Sigma-Aldrich, St. Louis, MO, USA) density gradient centrifugation $\left(1,000 \mathrm{~g}\right.$ for $30 \mathrm{~min}$ at $\left.4^{\circ} \mathrm{C}\right)$ was performed following heparinization (the heparin was also purchased from Sigma-Aldrich), and the intermediate monocyte layer was washed twice with phosphate-buffered saline (PBS) and inoculated into $75 \mathrm{~cm}^{2}$ flasks (Corning Incorporated, Corning, NY, USA) and cultured with L-Dulbecco's modified Eagle's medium (DMEM; Hyclone; GE Healthcare Life Sciences, Logan, UT, USA) with $10 \%$ fetal bovine serum (FBS; Hyclone; GE Healthcare Life Sciences) and penicillin-streptomycin (10,000 U/ml; Gibco; Thermo Fisher Scientific, Inc.). The media was changed after $72 \mathrm{~h}$, non-adherent cells were discarded, and the remaining adherent cells were subsequently verified by flow cytometry. The medium was changed every 2-4 days until the cells reached $90 \%$ confluence and then trypsin digestion and passaging was conducted (the trypsin was purchased from Sigma-Aldrich). The passage 3 (P3) MSCs were trypsinized, counted and single cell suspensions $\left(1 \times 10^{6} / \mathrm{ml}\right)$ were produced. Subsequently, $100-\mu 1$ aliquots of these suspensions were placed into microcentrifuge tubes with cluster of differentiation (CD)29 (cat. no. SAB4700394), CD44 (cat. no. SAB4700179), CD105 (cat. no. SAB4700262), CD34 (cat. no. SAB4700160) and CD45 (cat. no. SAB4700474) monoclonal mouse anti-human antibodies (all purchased from Sigma-Aldrich), incubated at room temperature for $30 \mathrm{~min}$, and analyzed on a Gallios ${ }^{\mathrm{TM}}$ flow cytometer (Beckman Coulter, Inc., Brea, CA, USA). All antibodies were used at a dilution of $1: 1,000$.

In vitro adipogenic differentiation and microarray expression. The MSCs from all the AIS patients and controls were cultured to $\mathrm{P}$, following which all cells were cultured in adipogenic solution [L-DMEM with $10 \% \mathrm{FBS}$ and $1 \mu \mathrm{mol} / 1 \mathrm{dexa}-$ methasone, $10 \mathrm{mg} / 1$ recombinant human insulin, $0.5 \mathrm{mmol} / 1$ 3-isobutyl-1-methylxanthine and $100 \mu \mathrm{mol} / 1$ indomethacin (all reagents except FBS were purchased from Sigma-Aldrich)] for 14 days. The cells were then stained with Oil red $\mathrm{O}(0.5 \mathrm{~g}$ in $100 \mathrm{ml}$ iso-propanol; Sigma-Aldrich).

Total RNA was extracted with TRIzol (Sigma-Aldrich) from adipogenic MSCs that had been cultured for 14 days. A Nanodrop 2000 spectrophotometer (Thermo Fisher Scientific, Inc.) was used to measure the optical density (OD) values (A260/A280) of the samples, and electrophoresis of the $28 \mathrm{~S} / 18 \mathrm{~S}$ bands was conducted to assess the quality of the total RNA samples. Following reverse transcription to cDNA (see next section), the AIS and control samples were labeled with Cy5 and Cy3 fluorescent tags, respectively. Hybridization experiments were performed with a cDNA direct labeling method [PrimeScript ${ }^{\mathrm{TM}}$ RT Reagent kit (Perfect Real Time), Takara Bio, Dalian, China] using the same quantity of probes, and hybridization was conducted at $45^{\circ} \mathrm{C}$ for $16 \mathrm{~h}$ using SYBR ${ }^{\circledR}$ Premix Ex Taq ${ }^{\mathrm{TM}}$ (Takara Bio).

Microarray hybridizations were conducted by the CapitalBio Corporation (Beijing, China). Data analysis was performed using the Significance Analysis of Microarray software (SAM 3.0, Stanford University, Stanford, CA, USA; http://statweb.stanford.edu/ tibs/SAM/). P-values were considered significant at a fold change of $\geq 2.0$ or $\leq 0.5$.

Semiquantitative RT-PCR. Semiquantitative RT-PCR was performed to validate the microarray results on P3 MSCs from 20 AIS patients and 10 healthy controls that had undergone adipogenic differentiation for 14 days. Total RNA was extracted from each sample using an E.Z.N.A ${ }^{\circledR}$ HP Total RNA Isolation kit (Omega Bio-Tek, Inc., Norcross, GA, USA). The reverse transcription to cDNA was performed on an $\mathrm{S} 1000^{\mathrm{TM}}$ thermal cycler $\left(15 \mathrm{~min}\right.$ at $37^{\circ} \mathrm{C}, 85^{\circ} \mathrm{C}$ for $5 \mathrm{sec}$; Bio-Rad Laboratories, Inc., Hercules, CA, USA) RNA concentration was determined and the samples were stored at $-20^{\circ} \mathrm{C}$. A Thermo Scientific RevertAid First Strand cDNA Synthesis kit (Thermo Fisher Scientific, Inc.) was used to perform reverse transcription. A spectral analyzer (Nanodrop 2000; Thermo Fisher Scientific, Inc.) was used to verify concentrations and the samples were stored at $-80^{\circ} \mathrm{C}$.

Seven genes (LGALS12, Spot14, MARC1, GPNMB, CTSK, IGFBP6 and NT5E; Table I) were selected for analysis of differential expression, and glyceraldehyde 3-phosphate dehydrogenase served as an internal reference. Semiquantitative RT-PCR was performed to quantify the expression levels of these seven genes to verify the accuracy of the microarray results. The specific primers used are presented in Table I and semiquantitative RT-PCR was conducted using the iQ5 PCR amplification system (Bio-Rad Laboratories, Inc.). Quantities of 50 ng cDNA (in $2 \mu \mathrm{l}$ ) were used, and the thermocycling conditions were: $95^{\circ} \mathrm{C}$ for $30 \mathrm{sec}$; 40 cycles of $95^{\circ} \mathrm{C}$ for $5 \mathrm{sec}, 60^{\circ} \mathrm{C}$ for $30 \mathrm{sec}$. Each sample was examined separately and in duplicate.

Western blotting. P3 MSCs from 5 AIS patients and 5 controls were cultured to promote adipogenic differentiation for 14 days. A protein extraction kit (Beyotime Institute of Biotechnology, Beijing, China) was used to extract total proteins. Briefly, the cells were washed with cold PBS twice and 5X cell lysis buffer (radioimmunoprecipitation assay buffer + phenylmethylsulfonyl fluoride) was added and incubated with the samples on ice for $30 \mathrm{~min}$. The samples were centrifuged at $12,000 \mathrm{x} \mathrm{g}$ and the supernatant was collected and the protein concentration was measured using the bicinchoninic acid kit of Sigma-Aldrich (50 $\mu \mathrm{g} / \mathrm{lane})$. Subsequently, the proteins were run on $10 \%$ sodium dodecyl sulfate-polyacrylamide gels at $40 \mathrm{~V}$ for $5 \mathrm{~h}$, and electrophoretically transferred to polyvinyldifluoride membranes. The blots were blocked in $50 \mathrm{~g} / 1$ skimmed milk for $2 \mathrm{~h}$ prior to incubation with rabbit anti-human Spot14 (or anti-THRSP; cat. no. HPA040642) or rabbit anti-human Spot 14-R (cat.no. HPA038816) monoclonal antibodies (dilution, 1:100; Atlas Antibodies AB, Stockholm, Sweden) at $4^{\circ} \mathrm{C}$ overnight. The blots were washed with TPS (Sigma-Aldrich), prior to incubation with mouse anti-rabbit secondary antibody (dilution, 1:1,000) at room temperature for $1 \mathrm{~h}$. Bands were visualized using diaminobenzidine (DAB; Sigma-Aldrich). A negative control was performed without 
Table I. Primer design of semiquantitative RT-PCR validation.

\begin{tabular}{llll}
\hline Gene symbol & Ref Seq ID & \multicolumn{1}{c}{ Forward primer } & \multicolumn{1}{c}{ Reverse primer } \\
\hline GPNMB & NM_002510 & 5'-CCTCGTGGGCTCAAATATAACAT-3' & 5'-ACTGTCCTCTGACCATGCTGT-3' \\
CTSK & NM_000396 & 5'-ACTCAAAGTACCCCTGTCTCAT-3' & 5'-CCACAGAGCTAAAAGCCCAAC-3' \\
IGFBP6 & NM_002178 & 5'-GAGGGGCTCAAACACTCTACG-3' & 5'-CCATCCGATCCACACACCA-3' \\
NT5E & NM_002526 & 5'-CCAGTACCAGGGCACTATCTG-3' & 5'-TGGCTCGATCAGTCCTTCCA-3' \\
MARC1 & NM_022746 & 5'-CCTCGCCTGGTCCTGATTTC-3' & 5'-GGCAGTAGTAGGTCCTTTGTGT-3' \\
LGALS12 & AF222694 & 5'-AACCCTCGCTTCCATACCAC-3' & 5'-TCCTCATTCCCGAAGAGAAAGAG-3' \\
Spot14 & AW272342 & 5'-CAGGTGCTAACCAAGCGTTAC-3' & 5'-CAGAAGGCTGGGGATCATCA-3'
\end{tabular}

Primer design of the genes identified on microarray that were randomly selected for RT-PCR validation. RT-PCR, reverse transcription-polymerase chain reaction; GPNMB, glycoprotein nmb; CTSK, cathepsin K; IGFBP6, insulin like growth factor binding protein 6; NT5E, 5'-nucleotidase ecto; MARC1, mitochondrial amidoxime reducing component 1; LGALS12, lectin, galactoside-binding, soluble, 12 ; Spot14 (or THRSP), thyroid hormone responsive.

the primary antibody. A LabWorks gel image analysis system (version 4.0; UVP, Inc., Upland, CA, USA) was used to scan the film and perform semiquantitative analysis.

Immunohistochemistry. Muscle and adipose tissue samples were taken from all 33 cases during surgery. Deparaffinized $4 \mu \mathrm{m}$ sections of paraffin-embedded specimens were placed in xylene twice and dehydrated with a series of alcohol [100, 95 and $80 \%$ ethanol/double-distilled $\mathrm{H}_{2} \mathrm{O}_{2}$ (v/v)], and rehydrated in PBS (the paraffin, xylene and ethanol were all purchased from Sigma-Aldrich). Tissue sections immersed in $0.01 \mathrm{M}$ sodium citrate buffer ( $\mathrm{pH}$ 6.0; BD Biosciences, Franklin Lakes, NJ, USA) in a microwave oven for antigen retrieval. Endogenous peroxidase was inactivated using 3\% hydrogen peroxide for $10 \mathrm{~min}$. Following rinsing with PBS, normal goat serum (BD Biosciences) was applied for $10 \mathrm{~min}$ to reduce non-specific staining. The sections were incubated with polyclonal primary rabbit antibodies against Spot14 at $4^{\circ} \mathrm{C}$ for $40 \mathrm{~min}$. Subsequent to washing with PBS for $5 \mathrm{~min}$, the sections were incubated for $10 \mathrm{~min}$ with biotinylated goat anti-rabbit immunoglobulins, washed with PBS and then incubated for 10 min with the streptavidin-horseradish peroxidase solution (Sigma-Aldrich), which binds to the biotin-labeled secondary antibody. Following PBS rinsing, sections were stained by incubation for 5 min with DAB in $\mathrm{H}_{2} \mathrm{O}_{2}$. The sections were washed in water and counterstained with hematoxylin (Sigma-Aldrich) for $3 \mathrm{~min}$. Negative controls for each biopsy were processed in the same manner, using PBS in place of the primary antibody. Healthy human adipose and muscle tissue samples were used as positive controls for Spot14.

Microscopy. Expression levels of Spot14 were determined using a visual grading system (Axio Imager Z1 microscope; Zeiss, Oberkochen, Germany) based on the extent of staining (percentage of positive cells graded on a scale from 0 to 4 : $0,<5 \% ; 1,5-25 \% ; 2,25-50 \% ; 3,50-75 \% ; 4,>75 \%$ ) and the intensity of staining (graded on a scale of $0-3$ : 0 , none; 1 , weak staining; 2 , moderate staining; 3 , strong staining).

Statistical analysis. Data were expressed as the mean \pm standard error. To analyze the microarray results, analyses of variance and with post-hoc Bonferroni tests were performed to compare the mean OD values from the AIS and control groups. Comparisons within groups were performed with paired t-tests (one-way analysis of variance was used for multiple comparisons). The expression levels analyzed by semiquantitative RT-PCR in AIS and control samples were analyzed using linear mixed model analysis. In addition, fold changes in relative expression levels from semiquantitative RT-PCR were calculated with $\chi^{2}$ analyses. $\mathrm{P}<0.05$ (two-sided) was considered to indicate a statistically significant difference and all statistical tests were conducted using SPSS version 13.0 software (SPSS Inc., Chicago, IL, USA).

\section{Results}

Isolation, culture, and differentiation of MSCs. Adherent cells were observed 1 day after inoculation of primary cells. They exhibited spindle or polygonal appearances with visible nuclei. Following culture for 14 days, the cells reached confluency. Microscopy confirmed that P3 cells were $~ 90 \%$ confluent with concentric growth and directional fusiform arrangement (magnification, x100; Fig. 1A and B). The P3 MSCs were adipogenically differentiated for 14 days, which induced morphological changes. The cells took on oval shapes, increased in volume, and developed round translucent droplets in the cytoplasm. Oil red $\mathrm{O}$ staining was strongly positive (Fig. 1C and D; magnification, x100). Flow cytometry was conducted to phenotypically identify MSCs. The results demonstrated that the cells were positive for CD29, CD44, and CD105 expression, while CD34 and CD45 expression was negative, confirming that they were MSCs (Fig. 1E and F).

Gene expression profiling. Differentially expressed genes (300) were identified between adipogenic MSCs from AIS patients and controls with a fold change $\geq 2.0$ or $\leq 0.5$ in the SAM output. In total, 111 and 189 genes were upregulated and downregulated, respectively, in cells from AIS patients. Fig. 2A and B presents the cluster and scatter plots of the microarray analysis, seven genes with the most marked differential expression were selected for verification (Table I). Upregulated mRNA expression of LGALS12, Spot14 and 

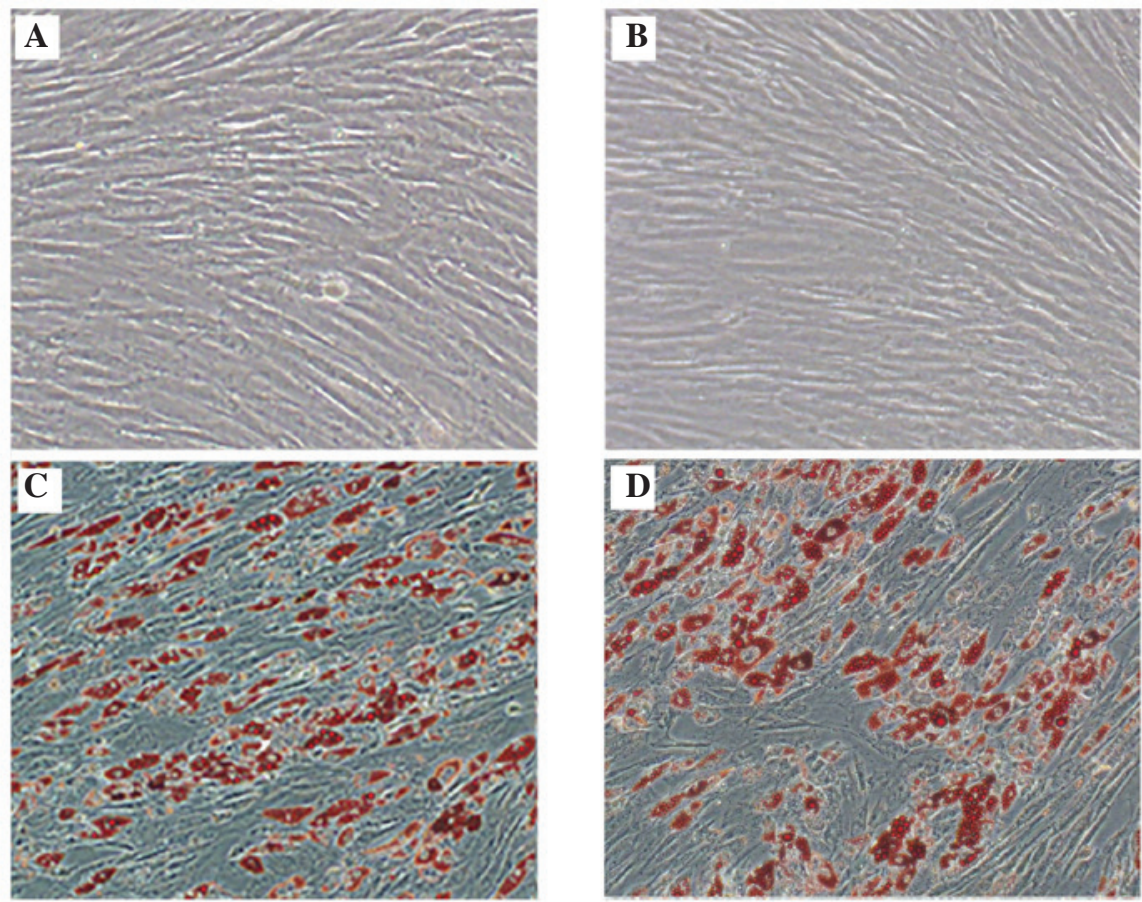

$\mathbf{E}$
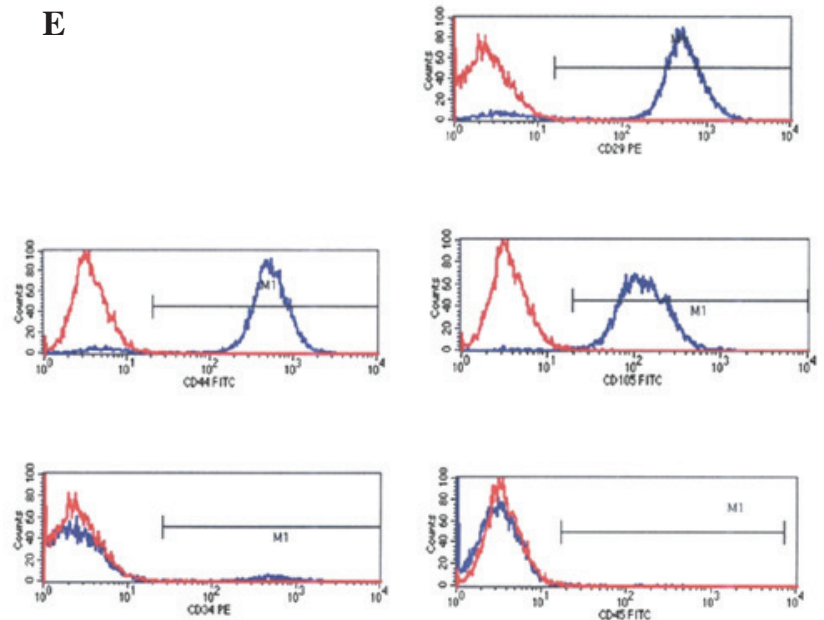

$\mathbf{F}$
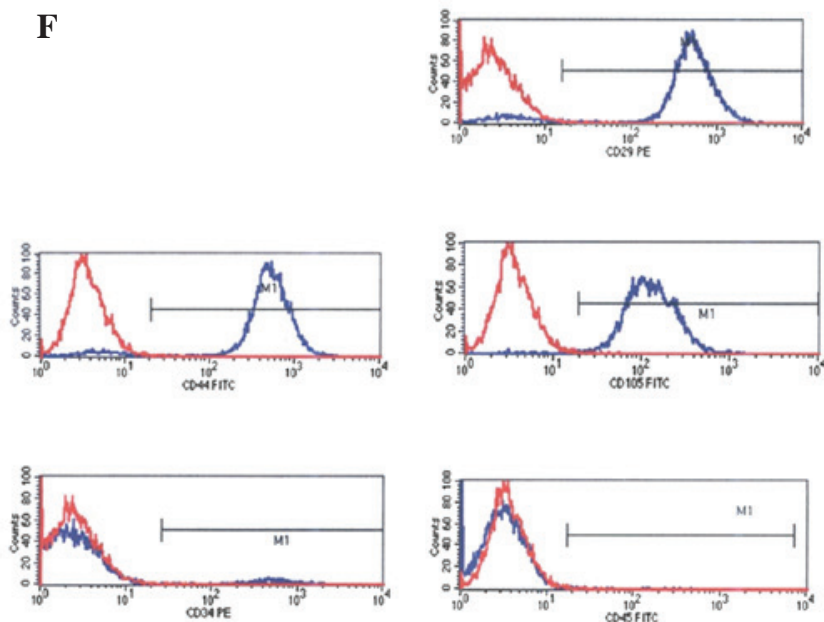

Figure 1. Morphological characteristics of MSCs and flow cytometry results. (A and B) Passage 3 MSCs from AIS patients and controls, respectively. (C and D) Adipogenic MSCs at 14 days stained with Oil red O. Magnification, x100. (E and F) Results from flow cytometric analysis. MSCs from AIS patients and control subjects, respectively. CD29, CD44, and CD105 expression were positive, while CD34 and CD45 expression were negative, demonstrating that the cells were MSCs. MSC, mesenchymal stem cell; AIS, adolescent idiopathic scoliosis; CD, cluster of differentiation.

MARC1 and downregulated mRNA expression of GPNMB, CTSK, IGFBP6 and NT5E was demonstrated by semiquantitative RT-PCR. Amplification was performed, and the melt curve profile demonstrated a single peak. The seven genes from microarray results were strongly confirmed (Fig. 2C), which indicated that microarray data was credible.

Protein expression levels of Spot 14 and S14R. The microarray results indicated that Spot14 gene expression levels were upregulated, and these were further assessed by western blot analysis. Fig. 3 demonstrates that Spot14 protein was highly expressed during adipogenic process of MSCs in AIS patients, suggesting that Spot14 may be involved in AIS pathogenesis. Protein expression levels of S14R were also investigated and demonstrated to be highly expressed in AIS patients.
The immunohistochemical staining demonstrated Spot14 was predominantly distributed in the cytoplasm and intercellular substances. Fig. 4A and B demonstrate the Spot14 immunostaining in representative patient and control samples in muscle tissue samples, and Fig. 4C and D present the results for the adipose tissue samples. In adipose tissue samples from AIS patients, the Spot14 positive ratios (based on the strength of staining) were significantly higher than in adipose tissue samples from the controls $(5.67: 1 ; \mathrm{P}<0.001)$. However, no significant difference was indicated in muscle tissue samples between AIS and the control. Spot14 was expressed in 85\% (17/20 cases) of the adipose tissue samples from AIS patients, but only $23.1 \%$ (3/13 cases) of adipose tissue from control exhibited Spot14 immunostaining. The results of Spot14 immunohistochemical staining are presented in Table II. 
A

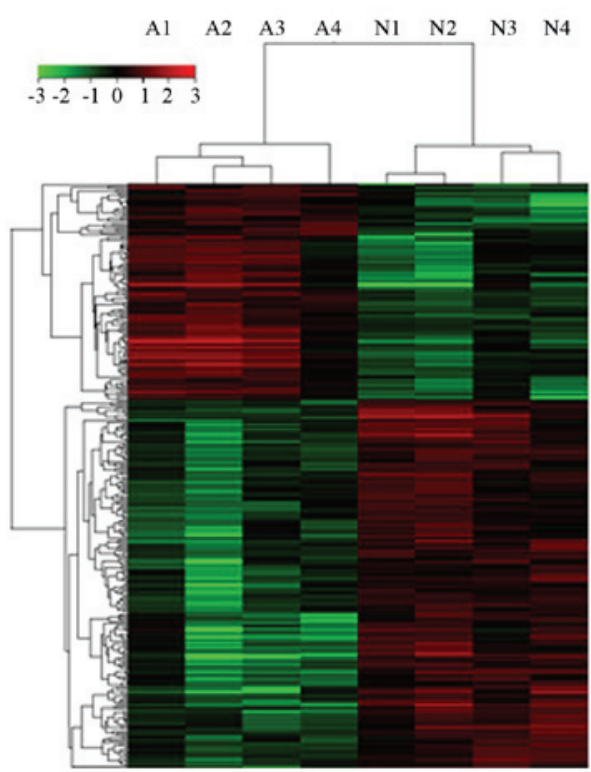

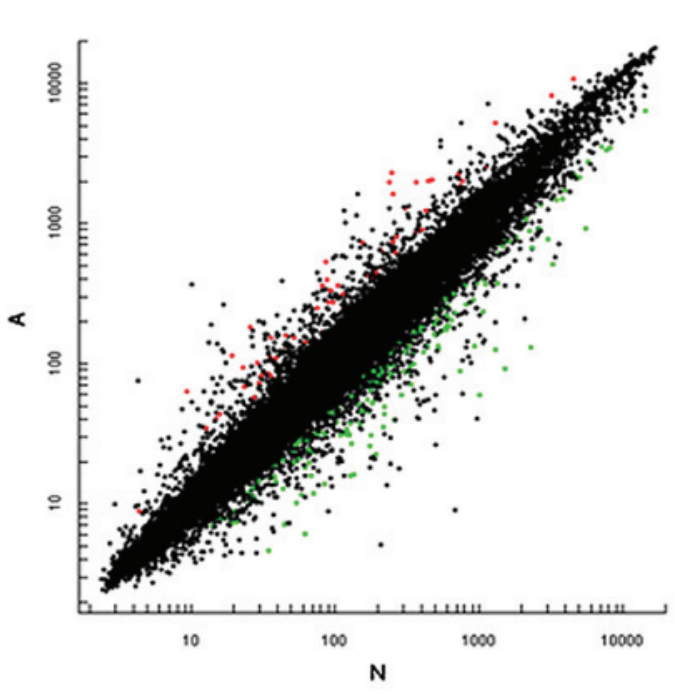

C

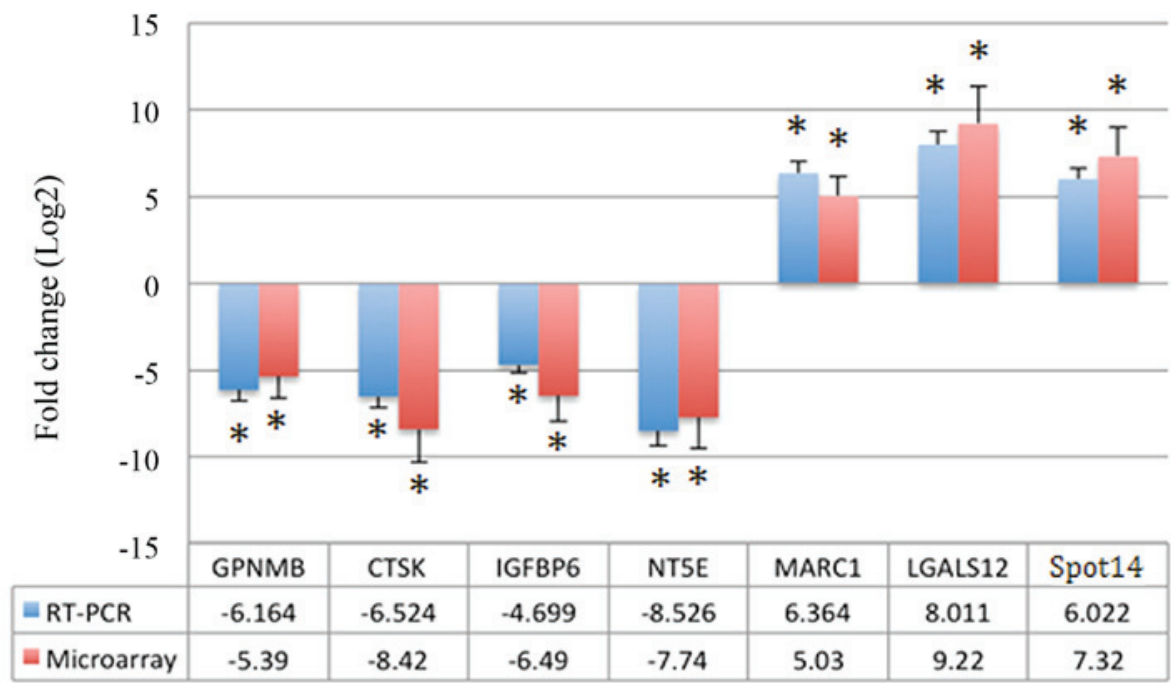

Figure 2. Microarray analysis results. (A) Cluster and (B) scatter plots of microarray data following Significance Analysis of Microarray analysis. In total, 300 genes were differentially expressed between AIS patients and control subjects. (C) The microarray results of selected genes and verification by RT-qPCR Differential gene expression between AIS patients and control subjects with fold changes $\geq 2.0$ or $\leq 0.05$ and RT-PCR validation, the two results indicate the same trend. "P<0.05 vs. the control cells. AIS, adolescent idiopathic sclerosis; RT-qPCR, reverse transcription-quantitative polymerase chain reaction; GPNMB, glycoprotein nmb; CTSK, cathepsin K; IGFBP6, insulin like growth factor binding protein 6; NT5E, 5'-nucleotidase ecto; MARC1, mitochondrial amidoxime reducing component 1; LGALS12, lectin, galactoside-binding, soluble, 12; THRSP, thyroid hormone responsive.

\section{Discussion}

To the best of our knowledge, this is the first study to investigate gene expression levels of MSC adipogenesis in AIS patients. MSC adipogenic differentiation was observed in AIS patients and healthy controls and microarray analysis was used to evaluate the differential gene expression of MSC adipogenesis in AIS. Furthermore, genes with marked differential expression were selected for analysis of protein expression and histological validation. Revealing Spot14 showed increased gene and protein expression in adipose tissues. Spot14 has an important regulatory role in the adipose differentiation process, the results from the present study demonstrate that there are significant gene and protein expression changes $(\mathrm{P}<0.001)$ in MSCs between AIS patients and healthy controls.
AIS is a complex disease and the underlying mechanism remains to be elucidated. Compared with healthy controls, AIS patients are taller, have lower BMI and decreased bone mass. The reasons for these differences are not fully elucidated, however, they may be associated with the gene regulation of MSC differentiation (5). Thus, the present study has important theoretical and clinical implications for the understanding of AIS pathogenesis, particularly regarding the role of MSC differentiation (8).

Previous studies of abnormal adipogenesis in AIS patients have demonstrated that leptin may be important in AIS pathogenesis $(11,12)$. Leptin is an important growth hormone that regulates female puberty, it is predominantly synthesized and secreted by adipose cells and is also involved in regulating appetite, increasing metabolism, and promoting energy and 
A

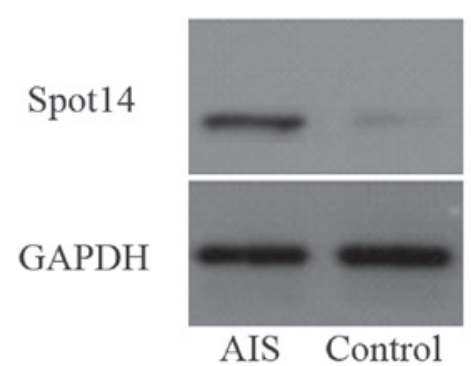

C

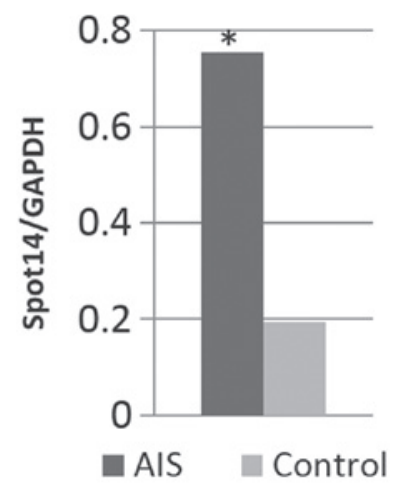

B

Spot14R

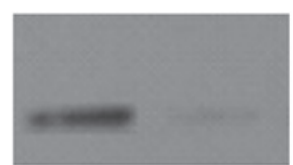

GAPDH

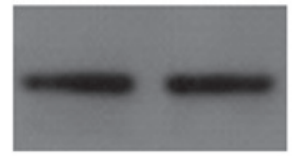

AIS Control

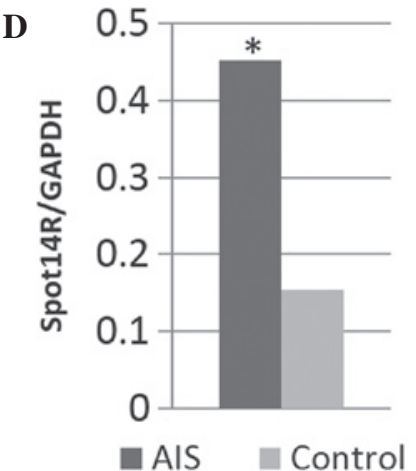

Figure 3. Western blotting results. Western blotting results demonstrating (A) Spot14 and (B) Spot14R expression of adipogenic MSCs harvested from AIS patients and control subjects. (C and D) Statistical analysis demonstrated a significant difference between the two groups. ${ }^{*} \mathrm{P}<0.05$.
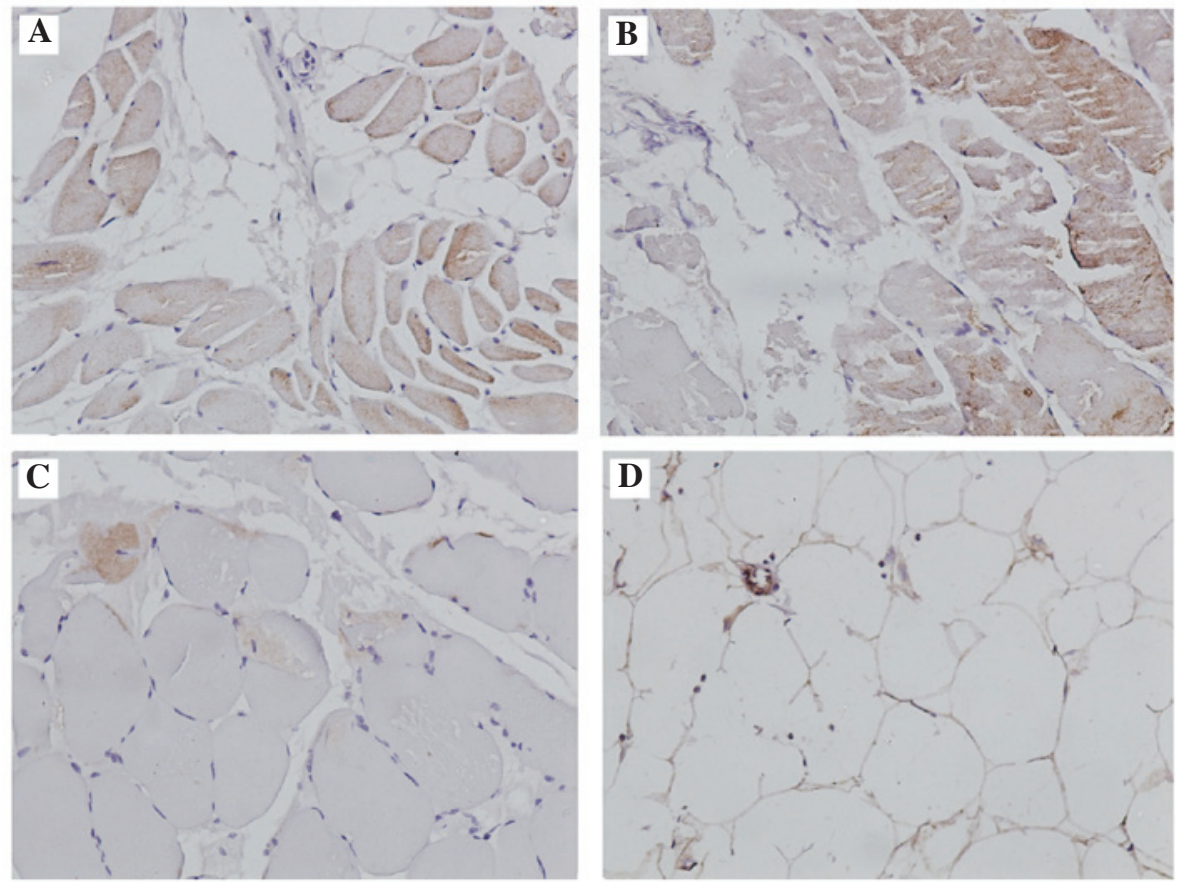

Figure 4. Immunohistochemistry. Immunohistochemical analysis for thyroid hormone-inducible nuclear protein expression in human (A) adipose and (B) muscle tissue samples from adolescent idiopathic scoliosis patients and (C) adipose and (D) muscle tissue samples from the control. Magnification, x200.

heat production. In addition, it is involved in the regulation of adolescent growth, angiogenesis, bone metabolism, and other processes. However, adipogenic differentiation regulation is a complex process involving numerous different signaling pathways. Spot14 is also a key lipogenesis factor (13). First identified in 1981, Spot14 mRNA rapidly responds to thyroid hormone. It has two subtypes, Spotl40t and Spotl4fl, with mRNA lengths of $\sim 797$ and $683 \mathrm{bp}$, respectively. The Spot14 gene is important in the regulation of lipid metabolism (14) and it is a transcription factor that controls adipogenesis enzyme activity; its predominant function is to control the transcription of genes for six enzymes involved in the adipogenic synthesis pathway. Mice lacking the Spot14 gene exhibit reduced de novo lipogenesis in the lactating mammary gland. However, no decrease in hepatic lipogenesis was observed in Spot14-null mice (15). It was hypothesized that this difference 
Table II. Immunohistochemistry analysis of Spot14.

\begin{tabular}{lccc}
\hline $\begin{array}{l}\text { Spot14 } \\
\text { positive case }\end{array}$ & P-value & $\begin{array}{c}\text { AIS patients } \\
(\%, \mathrm{n}=20)\end{array}$ & $\begin{array}{c}\text { Control } \\
(\%, \mathrm{n}=13)\end{array}$ \\
\hline Muscle & 0.313 & $3(15)$ & $2(15.4)$ \\
Adipose & $<0.001^{\mathrm{a}}$ & $17(85)$ & $3(23.1)$ \\
\hline
\end{tabular}

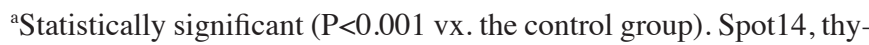
roid hormone-inducible nuclear protein; AIS, adolescent idiopathic scoliosis.

may be due to the expression of a paralogous gene designated S14R in the liver but not the mammary tissue. It is hypothesized that S14 and S14R interact during lipogenesis $(15,16)$.

A previous study demonstrated that the Spot14 gene is located at a chromosomal region associated with obesity (17), which may be associated with the low BMI of AIS patients. Results from the present study demonstrate that S14R was highly expressed at the mRNA and protein levels. It has been reported that the Spot14/S14R axis is involved in lipid metabolism and regulation of transcription by the RNA polymerase II promoter (13). The S14R gene is located on the X chromosome, which may be associated with the increased prevalence of AIS in females $(16,18)$. However, the biological functions and associated signaling pathways influenced by the Spot14/S14R axis in AIS patients remain to be elucidated.

A potential limitation of the present study was that all the experiments were performed to observe the differential expression and conduct validation. Future studies are required to investigate the functions of differential gene expression during MSC adipogenic differentiation in patients with AIS and an animal model should be used to verify the hypothesis from the present study.

In conclusion, in the present study MSC adipogenic differentiation in AIS patients and healthy controls was examined, with a specific focus on Spot14. This protein has been revealed to exert an important regulatory role in the adipose differentiation process, although the details concerning its involvement in AIS pathogenesis have yet to be fully elucidated. The present results demonstrate that there are significant gene and protein expression changes in MSCs from AIS patients compared with healthy controls. Future studies are required to explore the reasons for differential gene expression during MSC adipogenic differentiation in patients with AIS.

\section{Acknowledgements}

The authors would like to thank Professor Peng Xiang (Center for Stem Cell Biology and Tissue Engineering, Sun Yat-Sen University, Guangzhou, China) and Professor Xuenong Zou (The First Affiliated Hospital of Sun Yat-Sen University, Guangzhou, China) for their expert technical assistance and critical reading of this manuscript. The present study was supported by the National Natural Science Foundation of China (grant no. 81071439).

\section{References}

1. Lonstein JE: Adolescent idiopathic scoliosis. Lancet 344: 1407-1412, 1994

2. Weinstein SL, Dolan LA, Cheng JC, Danielsson A and Morcuende JA: Adolescent idiopathic scoliosis. Lancet 371: 1527-1537, 2008.

3. Weinstein SL: Adolescent idiopathic scoliosis: Prevalence and natural history. Instr Course Lect 38: 115-128, 1989.

4. Suh KT, Lee SS, Hwang SH, Kim SJ and Lee JS: Elevated soluble receptor activator of nuclear factor-kappaB ligand and reduced bone mineral density in patients with adolescent idiopathic scoliosis. Eur Spine J 16: 1563-1569, 2007.

5. Lee WT, Cheung CS, Tse YK, Guo X, Qin L, Ho SC, Lau J and Cheng JC: Generalized low bone mass of girls with adolescent idiopathic scoliosis is related to inadequate calcium intake and weight bearing physical activity in peripubertal period. Osteoporos Int 16: 1024-1035, 2005.

6. Cheng JC and Guo X: Osteopenia in adolescent idiopathic scoliosis. A primary problem or secondary to the spinal deformity? Spine (Phila Pa 1976) 22: 1716-1721, 1997.

7. Wang WJ, Hung VW, Lam TP, Ng BK, Qin L, Lee KM, Qiu Y, Cheng JC and Yeung HY: The association of disproportionate skeletal growth and abnormal radius dimension ratio with curve severity in adolescent idiopathic scoliosis. Eur Spine J 19: 726-731, 2010.

8. Zhuang Q, Li J, Wu Z, Zhang J, Sun W, Li T, Yan Y, Jiang Y, Zhao RC and Qiu G: Differential proteome analysis of bone marrow mesenchymal stem cells from adolescent idiopathic scoliosis patients. PLoS One 6: e18834, 2011.

9. Chu WC, Lam WM, Ng BK, Tze-Ping L, Lee KM, Guo X, Cheng JC, Burwell RG, Dangerfield PH and Jaspan T: Relative shortening and functional tethering of spinal cord in adolescent scoliosis-Result of asynchronous neuro-osseous growth, summary of an electronic focus group debate of the IBSE. Scoliosis 3: 8, 2008.

10. Nuttall ME and Gimble JM: Is there a therapeutic opportunity to either prevent or treat osteopenic disorders by inhibiting marrow adipogenesis? Bone 27: 177-184, 2000.

11. Liu Z, Tam EM, Sun GQ, Lam TP, Zhu ZZ, Sun X, Lee KM, Ng TB, Qiu Y, Cheng JC and Yeung HY: Abnormal leptin bioavailability in adolescent idiopathic scoliosis: An important new finding. Spine (Phila Pa 1976) 37: 599-604, 2012.

12. Liang G, Gao W, Liang A, Ye W, Peng Y, Zhang L, Sharma S, Su P and Huang D: Normal leptin expression, lower adipogenic ability, decreased leptin receptor and hyposensitivity to Leptin in Adolescent Idiopathic Scoliosis. PLoS One 7: e36648, 2012.

13. Colbert CL, Kim CW, Moon YA, Henry L, Palnitkar M, McKean WB, Fitzgerald K, Deisenhofer J, Horton JD and Kwon HJ: Crystal structure of Spot 14, a modulator of fatty acid synthesis. Proc Natl Acad Sci USA 107: 18820-18825, 2010.

14. Aipoalani DL, O'Callaghan BL, Mashek DG, Mariash CN and Towle HC: Overlapping roles of the glucose-responsive genes, S14 and S14R, in hepatic lipogenesis. Endocrinology 151: 2071-2077, 2010

15. Zhu Q, Mariash A, Margosian MR, Gopinath S, Fareed MT, Anderson GW and Mariash CN: Spot 14 gene deletion increases hepatic de novo lipogenesis. Endocrinology 142: 4363-4370, 2001.

16. Zhu Q, Anderson GW, Mucha GT, Parks EJ, Metkowski JK and Mariash CN: The Spot 14 protein is required for de novo lipid synthesis in the lactating mammary gland. Endocrinology 146: 3343-3350, 2005.

17. Bruford EA, Riise R, Teague PW, Porter K, Thomson KL, Moore AT, Jay M, Warburg M, Schinzel A, Tommerup N, et al: Linkage mapping in 29 Bardet-Biedl syndrome families confirms loci in chromosomal regions 11q13,15q22.3-q23 and 16q21. Genomics 41: 93-99, 1997.

18. Moreau A, Téruel C, Beylot M, Albalea V, Tamasi V, Umbdenstock T, Parmentier Y, Sa-Cunha A, Suc B, Fabre JM, et al: A novel pregnane X receptor and S14-mediated lipogenic pathway in human hepatocyte. Hepatology 49: 2068-2079, 2009. 